\title{
The interaction of versican with its binding partners
}

\author{
Yao Jiong WU ${ }^{1,3}$, David P. LA PIERRE ${ }^{1,3}$, Jin $\mathrm{WU}^{2}$, Albert J. YEE ${ }^{1}$, Burton B. YANG ${ }^{1,3, *}$ \\ ${ }^{1}$ Sunnybrook \& Women's College Health Sciences Centre, 2075 Bayview Avenue, Toronto M4N 3 M5 Canada \\ ${ }^{2}$ School of Chinese Medicine, The University of Hong Kong, Hong Kong SAR, China \\ ${ }^{3}$ Department of Laboratory Medicine and Pathobiology, University of Toronto, Canada
}

\begin{abstract}
Versican belongs to the family of the large aggregating chondroitin sulfate proteoglycans located primarily within the extracellular matrix (ECM). Versican, like other members of its family, has unique N- and C-terminal globular regions, each with multiple motifs. A large glycosaminoglycan-binding region lies between them. This review will begin by outlining these structures, in the context of ECM proteoglycans. The diverse binding partners afforded to versican by virtue of its modular design will then be examined. These include ECM components, such as hyaluronan, type I collagen, tenascin-R, fibulin-1, and -2, fibrillin-1, fibronectin, P- and L-selectins, and chemokines. Versican also binds to the cell surface proteins CD44, integrin $\beta 1$, epidermal growth factor receptor, and P-selectin glycoprotein ligand- 1 . These multiple interactors play important roles in cell behaviour, and the roles of versican in modulating such processes are discussed.
\end{abstract}

Keywords: extracellular matrix, proteoglycan, G3 domain, glycosaminoglycan, interaction.

\section{INTRODUCTION}

The extracellular matrix (ECM) is a highly-ordered supramacromolecular structure with many physiological and pathophysiological roles. The cells which produce matrix molecules are crucial in determining matrix function and metabolism. Conversely, and significantly, matrix components also have the ability to alter cell functions. The biochemical properties of a tissue are thus intimately related to the architecture of its matrix. The ECM comprises a variety of versatile collagen superfamily molecules and non-collagenous matrix molecules, including hyaluronan, proteoglycans, and glycoproteins, that are secreted locally and assemble into an organized meshwork. Proteoglycans are composed of core proteins covalently linked to long glycosaminoglycan (GAG) chains. Chondroitin sulfate proteoglycans and heparan sulfate proteoglycans are two large groups of proteoglycans. One

\footnotetext{
*Correspondence: Burton B. YANG

Research Building, Sunnybrook \& Women's College Health Sciences Centre, 2075 Bayview Avenue, Toronto M4N 3M5 Canada

Tel: +416-480-5874; Fax: +416-480-5737;

E-mail: Burton.Yang@sw.ca

Abbreviations: EGF (epidermal growth factor); CRD (carbohydrate recognition domain); CBP (complement binding protein).
}

sub-group of the former, the large aggregating chondroitin sulfate proteoglycans (which includes aggrecan, versican, neurocan and brevican), has been studied extensively [1-11].

\section{PROTEOGLYCANS: GENERAL STRUCTURE AND FUNCTIONS}

Recognized in the early 1960 s as important structural components of the ECM of cartilage, proteoglycans were once thought to be specific to that tissue. By now it has become clear that they are found in the ECM of all tissues. Proteoglycans have a much higher ratio of polysaccharide to protein than do collagen, fibronectin, and ECM glycoproteins. The polysaccharide chains in proteoglycans are long, repeating, linear polymers of specific GAG disaccharides. Proteoglycans may vary through differential expression of genes encoding core proteins, and different exon usage of these genes, as well as variations in the length, number, and types of GAG chains. Thus, a large and diverse array of proteoglycan binding partners and therefore functions exists.

Proteoglycans are present both in the ECM and on the cell surface of many cell types. A given ECM may contain various proteoglycans with several different types of core proteins and GAGs, which vary in number, length, and composition. As such, the molecular weight and charge 
density of a population of proteoglycans can be expressed only as an average; individual proteoglycans can be significantly different.

The common GAGs are heparin, heparan sulfate, and chondroitin sulfate. In proteoglycans, GAG polysaccharides are usually composed of many tandem repeats of a disaccharide pair: one sugar is usually an uronic acid and the other is either $\mathrm{N}$-acetyl-glucosamine or $\mathrm{N}$-acetylgalactosamine. One or both of the sugars contain one or two sulfate residues. Thus each GAG chain bears many negative charges, which makes it possible for proteoglycans to bind various positively charged molecules such as certain growth factors, cytokines, and chemokines [12]. Proteoglycans are usually named according to the structure of their principal repeating disaccharide of the attached GAG chains, which usually are added to the core proteins at serine residues through a sugar linker. However, the conformation of the core protein may be critically important in determining where the GAG chains link. The mechanism in controlling the length of GAGs remains unknown. GAG chains allow proteoglycans in the ECM or on the cell surface to generate immobilized gradients of certain growth factors and present them to their receptors; GAG chains also protect proteoglycans from proteolytic degradation $[13,14]$.

Members of the hyalectan family, so named for their ability to bind hyaluronan, are versican (also named PG-M), aggrecan, neurocan, and brevican [15]. The characteristic features of hyalectans, also termed lecticans, are their similar globular structures at both $\mathrm{N}$ - and C-termini [4, 16], and their lectin-like activity [17]. This sub-group of proteoglycans contains multiple domains and mediates a variety of cell activities [10, 18-22].

\section{STRUCTURE AND ISOFORMS OF VERSICAN}

Unlike aggrecan, which is almost exclusively expressed in cartilage and brain, and neurocan and brevican, which are proteoglycans of the central nervous system, versican is expressed in the ECM of a variety of tissues and organs, implying both importance and diversity in biological activities. The versican gene (CSPG2) has been localized to chromosome 13 in the murine genome and to chromosome 5 in the human genome [23]. Versican is encoded by 15 exons encompassing over 90 kilobase pairs of continuous DNA. Exon organization corresponds to the protein subdomains encoded by homologous proteins, with a remarkable conservation of exon size and intron phase. The versican promoter harbors a typical TATA box located approximately 16 base pairs upstream of the transcription start site, and binding sites for a number of transcription factors involved in regulation of gene expression. Stepwise 5 ' deletions have identified a strong enhancer

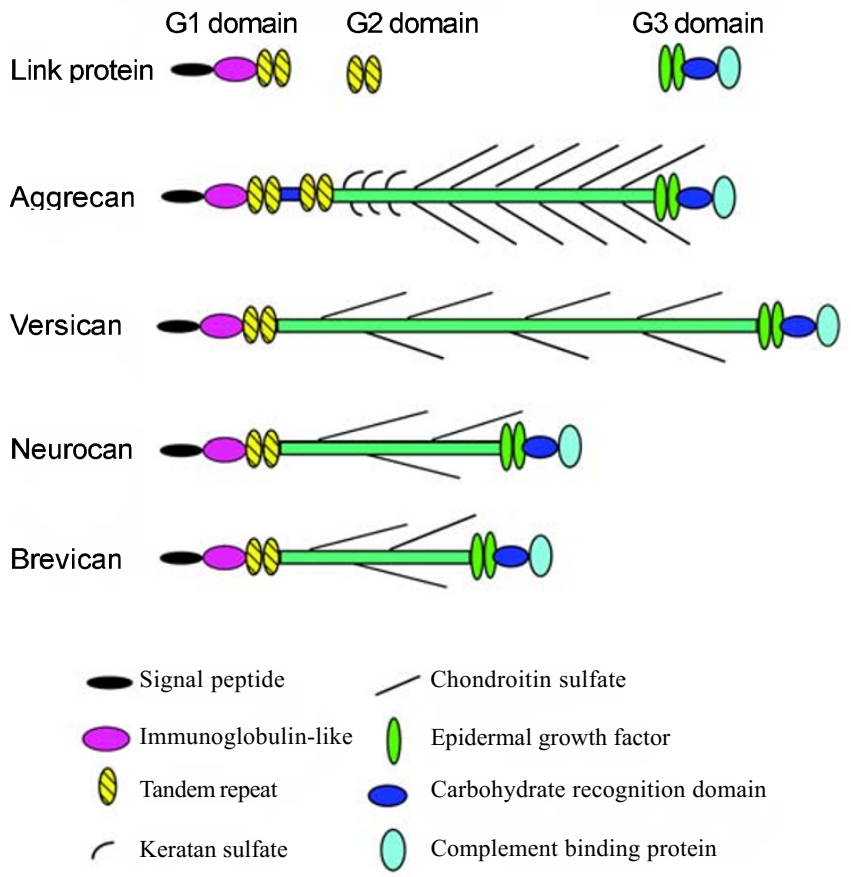

Fig. 1 Structure of versican isoforms. SP, signal peptide; Ig, immunoglobulin-like; TR, tandem repeat; EGF, epidermal growth factor; CS, chondroitin sulfate; $\mathrm{CRD}$, carbohydrate recognition domain; $\mathrm{CBP}$, complement binding protein.

element between -209 and -445 base pairs and a strong negative element between -445 and -632 base pairs [24].

Versican, along with the other hyalectans, consists of an N-terminal G1 domain, a C-terminal G3 domain, and CS chain binding regions between G1 and G3 (Fig. 1). The G1 domain is composed of an immunoglobular (Ig)like motif, followed by two proteoglycan tandem repeats that are known as HA-binding repeats (HABR). The G3 domain of versican consists of two epidermal growth factor (EGF)-like repeats, a carbohydrate recognition domain (CRD, also called the lectin-like motif), and a complement binding protein (CBP)-like motif. In this respect, G3 is similar to the selectin family [25] except that the lectican G3 domains contain only one complement binding protein-like subdomain, while selectins contain at least two. Alternative splicing of mRNA encoding GAG chain binding regions generates at least four isoforms of versican named V0, V1, V2 and V3 with molecular weight of the core proteins of about $370 \mathrm{kDa}, 263 \mathrm{kDa}, 180 \mathrm{kDa}$, and $74 \mathrm{kDa}$, respectively [2, 26-28]. Each contains different lengths of the GAG binding region with an accompanying variation in number of attached GAG chains (Fig. 1). V0, the largest versican isoform, contains two GAG binding regions called $\operatorname{CS} \alpha$ and $\operatorname{CS} \beta$ domains [26]. V1 isoform 
contains CS $\beta$ domain, and V2 isoform contains CS $\alpha$ domain. Versican V3 is solely composed of the G1 and G3 domains, lacking all potential GAG attachment sites [27].

In versican, the number of GAG side chains per length of the GAG-binding regions is rather constant. Estimated numbers of GAG chains are 17-23, 12-15, and 5-8 in versican isoforms V0, V1, and V2, respectively [26]. Furthermore, it seems rather unlikely that the smallest hyalectan, versican V3, carries GAG. Most of the above numbers are estimates based on putative carbohydrate attachment sites in the primary structures of the core proteins. However, the number, size, and composition of the carbohydrate substitution is not only influenced by the length of the GAG chain binding regions, but it may also be affected by G1 and G3 domains and the cell and tissue types. For instance, G1 inhibits but G3 promotes GAG chain attachment in aggrecan and versican [20, 29-31].

Versican's GAG profile varies amongst both tissues and specific molecules. GAG sulfation patterns change both markedly and precisely during chick brain development [32], indicating the significance of specific carbohydrate information. Versican GAG chains are long, repeating disaccharides of uronic acid, in the form of either glucouronic acid or idouronic acid, and $\mathrm{N}$-acetyl galactosamine. Three possible sulfation sites exist, leading to at least five specific chondroitin sulfate subtypes. Of these, C-4S and C$6 \mathrm{~S}$ are important mediators of molecular interaction. GAG chains are composed of approximately 40 repeating units. These chains repel each other, and within themselves, thus assuming long, extended shapes.

Versican's structure is complex, and its observed effects in vitro and in vivo are as well. It has been shown to be involved in diverse cell functions, such as adhesion, migration, proliferation, and apoptosis. Reports are, in fact, sometimes contradictory. The seemingly confused observations may be reconciled by an appreciation of the structure and expression diversity of versican. On the other hand, versican does not act by itself. Its many binding partners play important roles in determining the behaviours that involve versican. It falls, then, to dissecting molecular interactors of versican, which also vary spatially and temporally, in order to understand this fascinating proteoglycan's function.

It is clear from even a brief examination of versican's structure that this molecule has been aptly named. Different isoforms allow for GAG chain mutability both among cell types and within a discrete microenvironment. The many structural components that make up versican's core protein structure also lend a vast selection of ligands to its repertoire. As we shall see throughout the remainder of this review, versican's binding partners play many key roles in cellular function and set an essential place for versican in the minds of those wishing to understand the ECM.

\section{INTERACTION OF VERSICAN WITH OTHER MATRIX MOLECULES \\ Versican interaction with hyaluronan}

Hyaluronan is a large polysaccharide, composed of multiple repeats of the $\beta 1,3 \mathrm{~N}$-acetylglucosaminyl- $\beta 1,4$ glucuronide disaccharide unit, principally produced by stromal cells and deposited in the pericellular matrices and the ECM of most tissues. In the ECM, hyaluronan is bound to several proteins, helping to create a lattice that may regulate cell adhesion and migration [33, 34]. Hyaluronan production is increased at sites of cell proliferation, limb bud formation, inflammation, tissue remodelling, and tumor cell invasion. This molecule is thought to modulate cell behaviour by binding to specific cell surface receptors, most notably CD44 [35]. Consistent with this notion, CD44-hyaluronan interaction has been observed to enhance growth of certain tumors in vivo [35-37].

Hyaluronan interaction with CD44 on the cell surface has been intensively studied [38]. Hyaluronan-CD44 signaling can activate several pathways through activation of specific intermediates [39]. These include, but are not limited to, the Rho and Rac1 GTPases, whose activities lead to subsequent reorganization of the actin cytoskeleton [4042]; erbB2 tyrosine kinase [43], which leads to cell proliferation; src-related tyrosine kinases [44], and nuclear factor- $\mathrm{\kappa B}$ [NF- $\mathrm{\kappa B}][45,46]$. Hyaluronan and CD44 interaction have also been known to affect cell adhesion and migration. Incorporation of hyaluronan into Matrigel results in increased glioma cell invasion in vitro, and this effect is blocked by neutralizing antibodies to CD44 [47]. Versican has been found to be colocalized with hyaluronan, CD44, and tenascin in the pericellular matrix of cultured fibroblasts [48], and in epidermal keratinocyte tumours, levels of hyaluronan, CD44 and versican correlated with the aggressiveness of the disease [49]. Versican's binding to hyaluronan is well known, occurring through its tandem repeats (Fig. 2).

The association of lecticans with hyaluronan is, at least in part, mediated by link protein, and indeed it has recently been shown that both link protein and hyaluronan bind to versican's G1 domain [50]. Using different versican constructs, the authors showed that both HABR domains were required and sufficient for in vitro binding, but that binding affinity was increased when the Ig-like domain was included. Link protein contains two HABR domains which appear to interact with the homologous structures of versican, and this interaction did not affect versican's binding to HA. Overexpression of versican G1 domain can enhance cell proliferation and reduce cell adhesion in dif- 


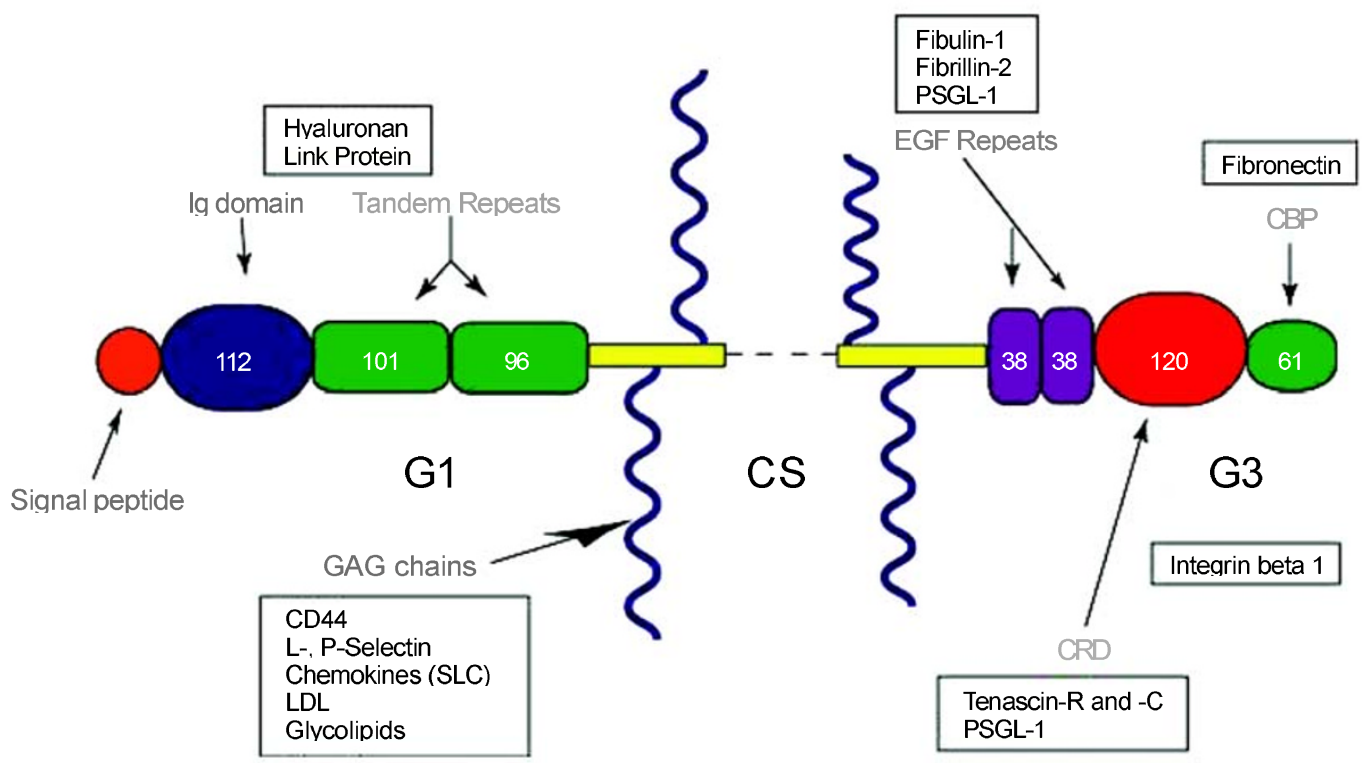

Fig. 2 The interaction of versican with other molecules. The locations of versican motifs that interact with other molecules are shown in the figure.

ferent cell types $[18,51]$. Taken together, studies have suggested that versican might enhance tumor behavior through interaction with hyaluronan and subsequent activation of CD44. Interestingly, studies have demonstrated that versican may interact directly with CD44 through its GAG chains, independent of hyaluronan [52]. It is important to know if this interaction also induces CD44 activation and transduces signals.

\section{Versican interaction with tenascin}

Tenascin-R binds to versican at its $\mathrm{C}$-terminal lectinlike domain [CRD] through protein-protein interactions [17] (Fig. 2). In addition to versican, tenascin binds to many other members of the lectican family, such as brevican [17], neurocan [53], phosphocan [53], and syndecan [54] through their CRD domains. The tenascin family of large ECM glycoproteins comprises five distinct genes coding for (in a chronological order) TN-C, TN-R, TN-X, TN-Y and TN-W [55-57]. Of these, only TN-R and $\mathrm{TN}-\mathrm{C}$ are expressed in the central nervous system. The structural homology shared by TN-R and TN-C has raised the logical question about similarities in function. Indeed, TN-R and TN-C both have been found to interfere with $\beta 1$ integrin-dependent cell adhesion and neurite outgrowth on fibronectin.

The defined spatiotemporal expression of chondroitin sulfate proteoglycans in the ECM or at the cell membrane of glial and neuronal cells is believed to play a role in biological phenomena as diverse as neural cell adhesion and migration; axon pathfinding, synaptogenesis and plasticity; growth factor and cytokine action; neuronal survival; and structural organization of the ECM [58]. Similar in distribution to chondroitin sulfate proteoglycans in the brain, TN-R is also implicated in such phenomena and therefore likely to interfere with chondroitin sulfate proteoglycan action or vice versa. TN-R has been suggested to participate in the macromolecular organization of perineuronal nets, along with hyaluronan and lecticans, based on its divalent cation-dependent homophilic binding properties [59].

This large hyaluronan matrix may create a local physical barrier, preventing other cells from entering the microenvironment [60]. TN-R binds to the C-terminal lectin domain of versican, its CRD, but surprisingly, this association is not mediated by carbohydrate moieties of TN-R [17]. Instead, protein-protein interactions, involving at least two fibronectin III domains of TN-R, are responsible. TN$\mathrm{R}$ gene knockout mice show abnormal perineuronal net formation [57], although the anatomy of all major brain areas and the formation and structure of myelin appear normal $[57,61]$. These results suggest that in the brain ECM, there may be many molecules, some of which may also interact with versican which are functionally complementary.

\section{Versican interaction with fibulins and fibrillin}

Fibulin-1 and fibulin-2 are modular ECM proteins, expressed in extracellular fibrils, basement membranes, and 
elastic fibres [62]. Versican binds to fibulin-2 [63] and fibrillin-1 [64] through its C-terminal lectin-like domain in a calcium-dependent manner. As with the unexpected observations with TN-R, versican appears to bind to fibulin1 , even following the latter's deglycosylation, again indicating protein-protein interaction [65]. The binding of fibulin-1 and fibulin-2 to versican has been mapped to their calcium-binding EGF-like domains (cbEGF). In addition to versican, aggrecan and brevican also interact with fibulin-2 by their lectin-like domain (Fig. 2), whereas neurocan does not [63]. Electron microscopy studies have confirmed the mapping and demonstrated that HA-aggrecan complexes can be cross-linked by the fibulins. The binding site for versican in microfibrils is most likely within a region of fibrillin-1, one of the major building blocks of microfibrils, between its cbEGF domains 11 and 21 [64]. Mutations in this region of the human gene, which potentially disrupt versican or other protein binding, can result in severe forms of Marfan syndrome ("neonatal" Marfan syndrome), with significant cardiovascular disease. The connection between versican and fibrillin microfibrils may thus be functionally significant, particularly in cardiovascular tissues. Fibulin may serve as a bridge between versican and fibrillin, forming highly ordered multimolecular structures important in the assembly of elastic fibers. This organization may be particularly meaningful in blood vessels and skin, where these molecules are co-localized [66].

\section{Versican interaction with fibronectin}

Studies have shown versican to interact with fibronectin, as well as collagen type I [67] and that these interactions are responsible for reduced cell adhesion in melanoma cells [68]. Fibronectin and collagen are integrin ligands and play roles in enhancing cell adhesion. Versican prevents cell binding to fibronectin, but not collagen type I, in several CNS cell types [69]. Conditioned medium (CM) from prostate cancer fibroblasts, containing versican V0 and V1, can result in reduced adhesion of prostate cancer cell lines to fibronectin, but not to laminin [70]. Adding RGD peptide, a classical integrin-binding sequence, to the V0/V1-containing CM yielded increased cell attachment to fibronectin. This may mean that versican can sequester fibronectin through fibronectin's RGD domain, preventing it from binding to cells. Treatment of the CM with ChABC, a chondroitinase, decreased versican's capacity to reduce adhesion suggesting that GAG side chains are involved.

Recently, we have demonstrated that versican G3 domain enhances tumor growth and angiogenesis, perhaps through enhancement of fibronectin expression [71]. We then investigated the possibility that the G3 domain interacts with fibronectin. Our results showed unambiguously that G3 interacts with fibronectin (Fig. 2). We have shown that the G3 domain directly binds to fibronectin using coimmunoprecipitation of expressed G3 with endogenous fibronectin, a pull-down experiment using His-tag affinity column, and co-immunoprecipitation of endogenous G3containing fragments with fibronectin. Native gel electrophoresis also demonstrated that versican and fibronectin co-migrated in samples extracted from human tumor tissues. Immunohistochemical analysis exhibited high expression of these three molecules in human brain tumors. Furthermore, versican G3 domain can form complexes with fibronectin and VEGF. The complex was found to stimulate endothelial cell adhesion, proliferation and migration. Removal of this complex with anti-fibronectin antibody reversed G3's enhancing effects on endothelial cell activities. Our results are in agreement with a recent publication showing that fibronectin binds to VEGF and increases the function of VEGF in endothelial cell migration in culture [72].

\section{INTERACTION OF VERSICAN WITH CELL SURFACE PROTEINS \\ Versican interaction with selectins and chemokines}

Cell surface proteins, L-, E, and P-selectin, contain an $\mathrm{N}$ terminal C-type lectin repeat, an EGF-like repeat, and a series of short consensus repeats, a single transmembrane sequence, and a cytoplasmic tail. As versican's CRD- and EGF-containing G3 domain is quite similar to extracellular selectin, it is only fitting that the two molecules interact. The binding of versican with P-selectin and L-selectin is strongly inhibited by the chondroitinase digestion of versican or by the addition of specific soluble chondroitin sulphites (CS) B, CS E, or heparan sulfate. Thus, versican appears to interact with selectins via its GAGs (Fig. 2), and may only do so when they are oversulfated [73]. Specific tetrasaccharides involved in the interaction were identified. In addition, versican is also found to bind certain chemokines and regulate chemokine function [74]. In particular, the chemokine SLC (secondary lymphoid tissue chemokine) was shown to bind to CS-B with strong specificity [74]. SLC assists in the binding of integrin $\alpha 4 \beta 7$, on leukocytes, with its ligand, MAdCAM-1, found on the endothelium [75]. Versican, through its GAG chains, may thus prevent leukocyte adhesion to vessel walls through SLC sequestration. Importantly, SLC binding to its receptor was not affected following GAG addition, suggesting that versican's negative modulation occurs downstream in the signal transduction pathway.

Although no consensus sequence has been identified in versican-binding chemokines, it appears that versican generally interacts with those that attract mononuclear lymphocytes. A specific layer of binding specificity is the particular CS chain that is expressed by versican, either 
through isoform expression or carbohydrate modification [74]. As the same GAG chains also bind L-selectin, and as some versican populations do not bind to this leukocyte cell surface adhesion molecule [76], versican expression patterns may be important in certain in vivo situations such as in inflammatory responses, where L-selectin and chemokines are concomitantly involved. Versican has been found to be upregulated in many inflammatory conditions, such as arthritis, asthma, and granulomatous lung diseases $[77,78]$. Therefore, versican might be involved in inflammation by regulating interaction with selectins and chemokine activities.

\section{Versican interaction with CD44}

CD44 is a type I transmembrane chondroitin sulfate proteoglycan. It is composed of a large extracellular domain containing a hyaluronan-binding region and 4 consensus sites for attachment of chondroitin sulfate, a 21amino acid transmembrane domain, and a 72-amino acid cytoplasmic tail [79]. CD44 can be altered by differential splicing of at least 10 variable exons encoding a segment of the extracellular domain, termed exons v1-v10, and by cell type-specific glycosylation [79]. CD44 has been shown to mediate cell-cell and cell-ECM interactions [80, 81], augment tumor cell motility on hyaluronan-coated substrates [81, 82], costimulate lymphocyte activation and tissue infiltration [83], and promote growth and metastasis of some tumor types $[84,85]$. Although GAG chains associated with some CD44 isoforms can also bind a subset of heparin-binding growth factors, cytokines, and ECM proteins such as fibronectin, most of the functions ascribed to CD44 can be attributed to its ability to bind to and internalize hyaluronan $[38,86]$.

The GAG chains of versican also bind to CD44 (Fig. 2), and competitive inhibition assays showed that versican, hyaluronan, and GAG chains all bind to the link module of CD44, with hyaluronan showing a substantially higher binding affinity. Although, as mentioned, versican GAG

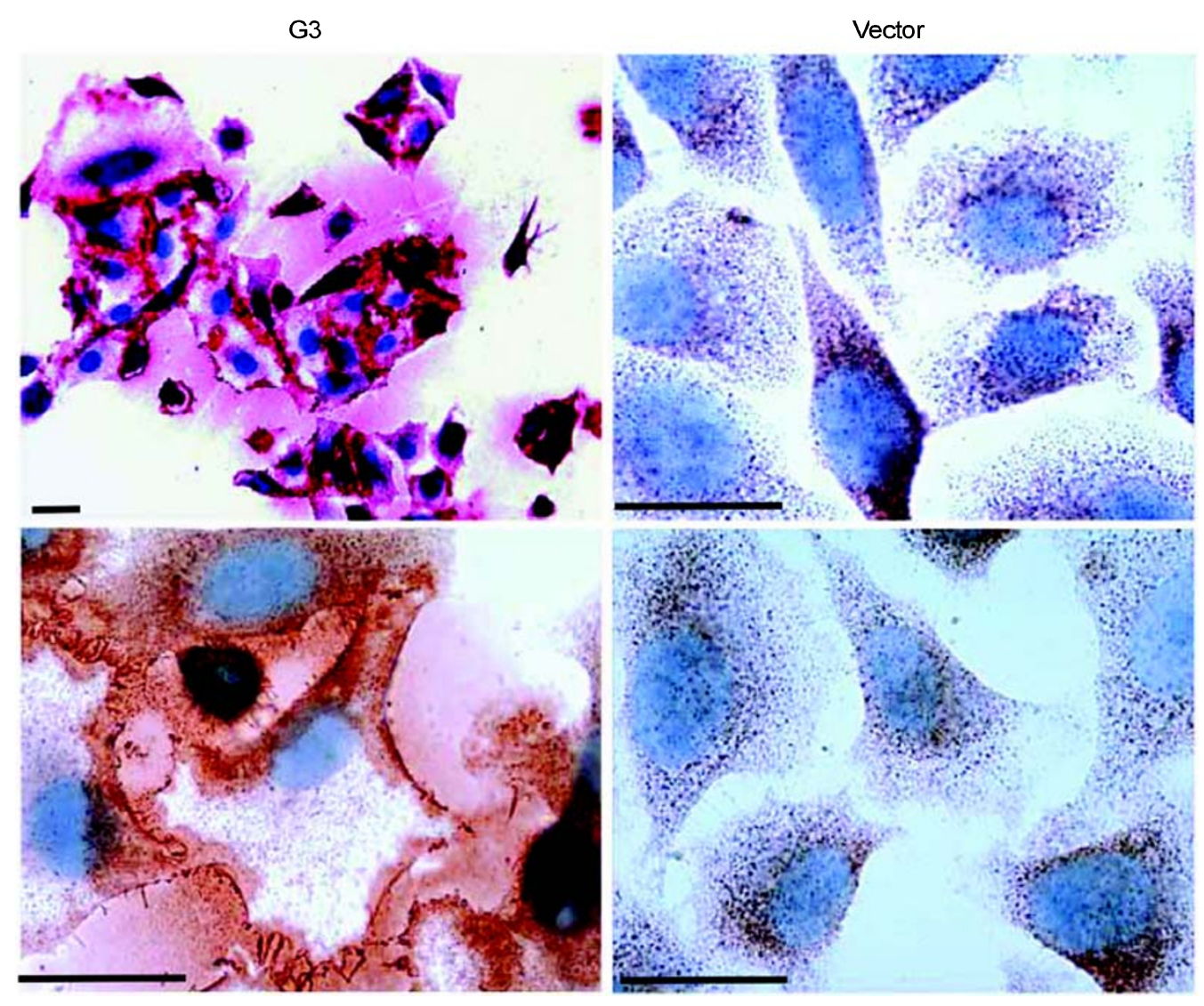

Fig. 3 Interactions of G3 with the cell surface. G3- and vector-transfected MT-1 cells were seeded on tissue culture plates to subconfluence. The cultures were fixed with $4 \%$ paraformaldehyde and probed with monoclonal antibody $4 \mathrm{~B} 6$ that recognizes an epitope engineered to the $\mathrm{G} 3$ construct. The immunostained cells were examined with a light microscope. G3 product was detected in the ECM and on the cell surface surrounding the cells. (scale bar=50 $\mu \mathrm{m}$ ) 
oversulfation is critical for binding to L- and P-selectins, GAG modifications do not appear to be important in CD44 binding. Interestingly, the binding of either L- or P-selectin to versican was inhibited with the addition of the solubilized other, but CD44 binding was not affected in the presence of selectins. This suggests that different GAG sites are responsible for these interactions [73].

As mentioned above, versican has been found to be colocalized with hyaluronan, CD44 and tenascin in the pericellular matrix of cultured fibroblasts [48]. As CD44 binds to both hyaluronan and versican, it is possible that the three may form complexes together. However, as versican and CD44 bind following hyaluronidase treatment, the complex association does not depend on the presence of hyaluronan [73].

The fact that GAG chains alone can bind the proteins mentioned here, without any assistance from versican core proteins, suggests that other proteoglycans that contain the proper side chains may also be involved in complexes. Many of the above-mentioned studies do, in fact, examine cohorts of chondroitin sulfate proteoglycans, with often similar, yet not identical, results seen among them.

\section{Versican interaction with integrin}

Integrins are important cell surface receptors, functioning as noncovalently linked heterodimers with $\alpha$ and $\beta$ subunits. Each $\alpha \beta$ combination has its own binding specificity and signaling properties. Although integrins were originally characterized as a family of cell surface receptors that are responsible for anchoring cells to the ECM, they have recently been shown to impact dynamic processes in normal and tumor cells, such as intracellular signaling and gene expression leading to cell migration, proliferation, differentiation, and survival $[87,88]$. Integrins can signal through the cell membrane in either direction: the extracellular binding activity of integrins is regulated from the inside of the cell, while the binding of the ECM elicits signals that are transmitted into the cell [88]. Integrins activate growth-promoting signaling pathways that are responsible for the anchorage requirement. Most integrins recognize several ECM proteins. For example, integrin $\beta 1$ pairs with various $\alpha$ subunits to bind a variety of ECM molecules, such as fibronectin, laminins, collagens, and vitronectin, osteopontin [89], nephronectin [90] and tenascin [91].

While versican also interacts with at least three of these proteins, we have found that it can also bind to integrin $\beta 1$ through its C-terminal G3 domain [92] (Fig. 2). As a result, the versican $\mathrm{G} 3$ product was detected not only in the ECM, but also on the cell surface surrounding the cells (Fig. 3). Interestingly, and surprisingly, versican does not contain an RGD motif, suggesting an entirely new mechanism of integrin binding. Astrocytoma cells expressing a construct containing versican CRD and CBP domains exhibited enhanced spreading and adhesion, with increased phosphorylation of focal adhesion kinase (FAK) and reduced $\mathrm{H}_{2} \mathrm{O}_{2}$ induced apoptosis. Exogenous addition of purified versican G3 product (containing two EGF-like motifs, CRD domain and CBP domain) was also able to induce FAK phosphorylation and increased cell adhesion.

\section{Versican interaction with epidermal growth factor receptor (EGFR)}

EGFR, another important cell surface receptor, mediates many cellular responses in both normal biological processes and pathological states. Like all receptor tyrosine kinases (RTKs), the EGFR family members comprise an extracellular domain containing a ligand-binding site, a single hydrophobic transmembrane helix, and a cytosolic domain that includes a conserved protein tyrosine kinase (PTK) core, flanked by regulatory sequences. The ligands of EGFR, such as EGF, bind to their receptors and induce conformational changes in the receptor monomers that promote their activation.

Integrins are able to form clusters with EGF receptors, and this physical interaction frequently affects the intensity of EGFR-induced down-stream signal to extracellular signal-regulated kinase (ERK) [93]. Growing evidence indicates that collaborative pathways derived from these two signals are crucial in regulating a range of cell activities, such as proliferation, differentiation, apoptosis, adhesion, and migration [93-96].

Astrocytoma cells expressing the C-terminal domain of versican exhibit reduced EGFR activity, represented by lower levels of EGF-induced EGFR autophosphorylation and accelerated reduction of the protein expression level after phosphorylation [97]. With enhanced integrin $\beta 1$ mediated adhesion signals, the cells exhibited increased integrin $\beta 1$-EGFR interaction, accompanied by increased FAK phosphorylation [92]. This increased cell adhesion and decreased EGF-induced EGFR phosphorylation was followed by reduced cell migration (Fig. 4).

The effects of versican on integrin and EGFR activation were also demonstrated in versican V1-transfected PC12 cells, a phaeochromocytoma cell line. Expression of versican V1 in PC12 cells resulted in upregulation of EGFR and integrin expression and induced NGF-independent PC12 cell neuronal differentiation and neurite outgrowth. Blockade of integrin $\beta 1$, EGFR, or Src, a downstream signal transduction kinase, significantly blocked this differentiation. This suggested that the differentiation requires signals mediated by versican derived from both integrin and EGFR [98]. Versican appears to not only present or recruit molecules to the cell surface, 


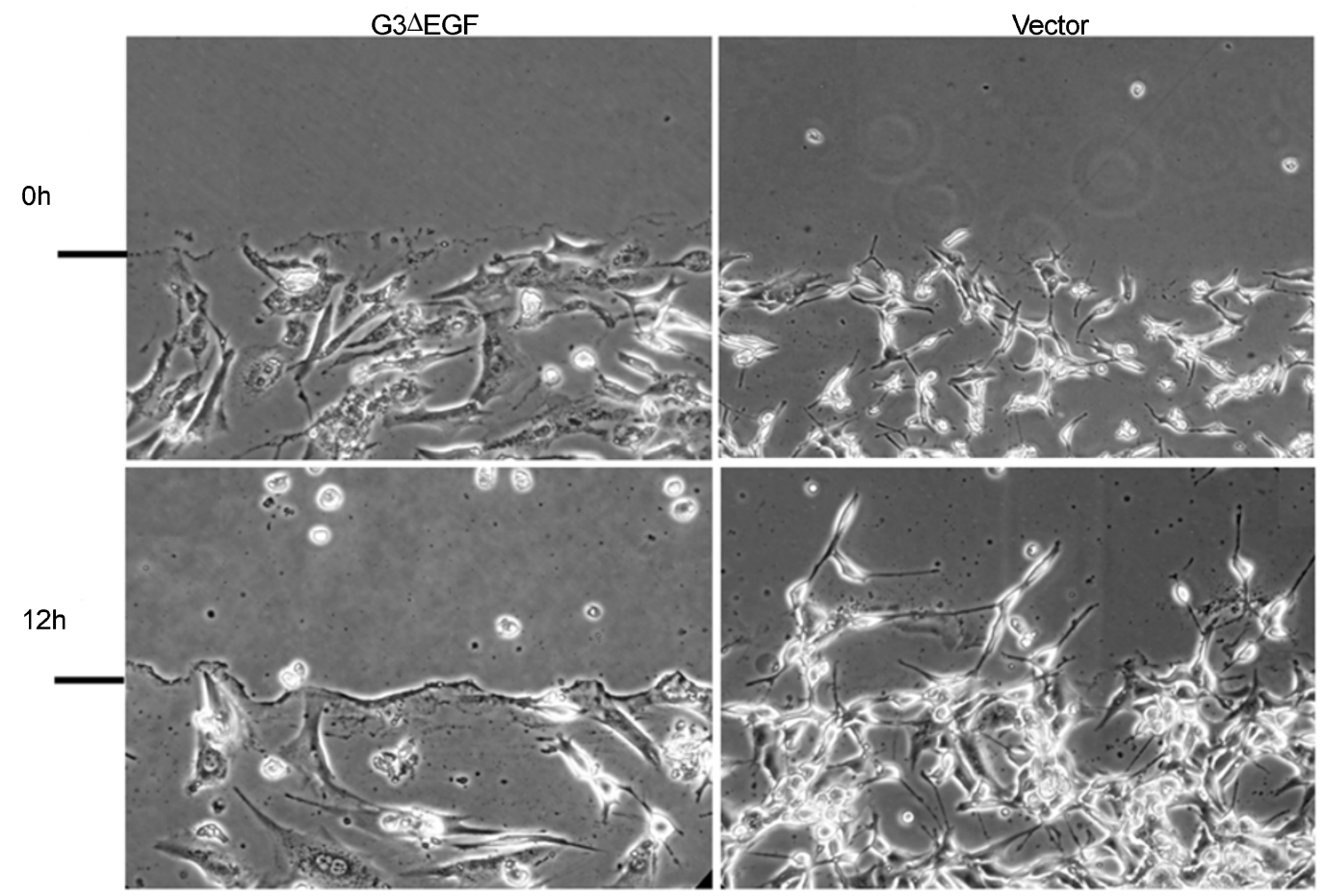

Fig. 4 Effect of G3 3 EGF expression on cell migration. Cells were cultured on tissue culture plates in DMEM containing $10 \%$ FBS to subconfluence. Parts of the cultures were removed by micro-tips. Cell migration was monitored for $24 \mathrm{~h}$. Vector-transfected cells exhibited a higher degree of locomotion than did the G3 $\Delta$ EGF-transfected cells.

but also to modulate the expression levels of genes and coordinate complex signal pathways.

\section{Versican interaction with P-selectin glycoprotein ligand-1}

P-selectin glycoprotein ligand-1 (PSGL-1) is a homodimeric glycoprotein held together by disulfide-bonds expressed on the cell surface of leukocytes and mediates leukocyte rolling on the vascular endothelium [99, 100]. Leukocyte rolling from the blood stream into tissues in response to inflammatory stimuli is a critical component of immunoresponse, which involves the activities of a range of adhesion and signaling molecules. The first events in this process are leukocyte adhesion and leukocyte rolling on the endothelial surface under vascular shear flow, mediated by selectins. PSGL-1 is expressed by essentially all blood leukocytes including lymphocytes, monocytes, neutrophils, and platelets, and has been shown to mediate the rolling of human neutrophils on selectins [101]. The N-terminal fragment of PSGL-1 is extensively glycosylated, which is critical for binding to the selectins [102]. PSGL1 binds to selectins and mediates leukocyte rolling on the vascular endothelium. We have demonstrated that PSGL1 binds to the C-terminal (G3 domain) of versican [103].
Cells transfected with PSGL-1 or a shorter form of PSGL1 containing the G3-binding site, or cells expressing endogenous PSGL-1, aggregate in the presence of versican or $\mathrm{G} 3$ product. The aggregation appears to be induced by G3 multimers that bind to PSGL-1 and form a network. Endogenous versican and/or G3-containing fragments also bind to PSGL-1 in human plasma. Removal of the endogenous G3-containing fragments reduces the effect of plasma on leukocyte aggregation. Our studies thus reveal a physiologically relevant role for PSGL-1/versican binding and may have implications in the immunoresponse [103].

\section{VERSICAN AND LDL}

Versican, along with other vascular proteoglycans, has been shown to bind to and retain low density lipoproteins (LDL) in vascular walls (Fig. 2), perhaps contributing to atherosclerotic formations [104]. Strong co-localization of LDL and versican has been observed. Lipoprotein lipase (LPL) has domains that bind to both LDL and proteoglycans, while oxidized LDL does not appear to efficiently interact with proteoglycans, including versican. LPL may act as an efficient bridge between these molecules [105]. It appears that the negatively charged GAG chains of versican 
interact with positively charged clusters of amino acids on apolipoproteins [104]. Increased LDL binding affinity is seen with longer GAG side chains, greater levels of sulfation, and increased ratios of $\mathrm{C} 6 \mathrm{~S}$ to $\mathrm{C} 4 \mathrm{~S}$ (or CS C to CS A) [106]. The number and composition of GAG chains in versican isoforms should have a significant effect on these molecular interactions. The interaction of versican with hyaluronan, which generates a very high local density of GAG chains, would also magnify this effect.

\section{CONCLUDING REMARKS}

The literature on versican's function can appear confusing and even contradictory. Versican has been reported to increase or decrease proliferation, migration or adhesion depending on the study approach. These issues begin to be understood with an appreciation for the isoforms of versican, its modular structure, and the varying binding partners that are thus involved. This, coupled with cell and tissue type-specific active signalling pathways, means that versican is almost dazzlingly flexible in its range of biological roles. The task of integrating versican - both spatially and temporally - into its web of interactors is daunting and demanding. It will no doubt also be very rewarding for both researchers and those whom this knowledge will benefit.

\section{ACKNOWLEDGEMENTS}

Our studies were supported by grants from National Sciences and Engineering Research Council of Canada (227937-01] and Canadian Institutes of Health Research (MOP-62729 and MOP-74469] to Burton B YANG.

\section{REFERENCES}

1 Kimata K, Oike Y, Tani K, et al. A large chondroitin sulfate proteoglycan (PG-M) synthesized before chondrogenesis in the limb bud of chick embryo. J Biol Chem 1986; 261:13517-25.

2 Shinomura T, Nishida Y, Ito K, et al. cDNA cloning of PG-M, a large chondroitin sulfate proteoglycan expressed during chondrogenesis in chick limb buds. Alternative spliced multiforms of PG-M and their relationships to versican. J Biol Chem 1993; 268:14461-9.

3 Zimmermann DR, Ruoslahti E. Multiple domains of the large fibroblast proteoglycan, versican. EMBO J 1989; 8:2975-81.

4 Kiani C, Chen L, Wu YJ, et al. Structure and function of aggrecan. Cell Res 2002; 12:19-32.

5 Li H, Schwartz NB, Vertel BM. cDNA cloning of chick cartilage chondroitin sulfate (aggrecan) core protein and identification of a stop codon in the aggrecan gene associated with the chondrodystrophy, nanomelia. J Biol Chem 1993; 268:2350411.

6 Rauch U, Karthikeyan L, Maurel P, et al. Cloning and primary structure of neurocan, a developmentally regulated, aggregating chondroitin sulfate proteoglycan of brain. J Biol Chem 1992; 267:19536-47.
7 Yamada H, Watanabe K, Shimonaka M, et al. Molecular cloning of brevican, a novel brain proteoglycan of the aggrecan/versican family. J Biol Chem 1994; 269:10119-26.

$8 \mathrm{Wu}$ Y, Zhang Y, Cao L, et al. Identification of the motif in versican G3 domain that plays a dominant-negative effect on astrocytoma cell proliferation through inhibiting versican secretion and binding. J Biol Chem 2001; 276:14178-86.

9 Zhang Y, Cao L, Kiani CG, et al. The G3 domain of versican inhibits mesenchymal chondrogenesis via the epidermal growth factor-like motifs. J Biol Chem 1998; 273:33054-63.

10 Zhang Y, Wu Y, Cao L, et al. Versican modulates embryonic chondrocyte morphology via the epidermal growth factor-like motifs in G3. Exp Cell Res 2001; 263:33-42.

11 Zhang Y, Cao L, Yang BL, et al. The G3 domain of versican enhances cell proliferation via epidermial growth factor-like motifs. J Biol Chem 1998; 273:21342-51.

12 Spivak-Kroizman T, Lemmon MA, Dikic I, et al. Heparin-induced oligomerization of FGF molecules is responsible for FGF receptor dimerization, activation, and cell proliferation. Cell 1994; 79:1015-24.

13 Ruoslahti E, Yamaguchi Y. Proteoglycans as modulators of growth factor activities. Cell 1991; 64:867-9.

14 Tanaka Y, Adams DH, Shaw S. Proteoglycans on endothelial cells present adhesion-inducing cytokines to leukocytes. Immunol Today 1993; 14:111-5.

15 Bandtlow CE, Zimmermann DR. Proteoglycans in the developing brain: new conceptual insights for old proteins. Physiol Rev 2000; 80:1267-90.

16 Retzler C, Wiedemann H, Kulbe G, et al. Structural and electron microscopic analysis of neurocan and recombinant neurocan fragments. J Biol Chem 1996; 271:17107-13.

17 Aspberg A, Miura R, Bourdoulous S, et al. The C-type lectin domains of lecticans, a family of aggregating chondroitin sulfate proteoglycans, bind tenascin-R by protein-protein interactions independent of carbohydrate moiety. Proc Natl Acad Sci U S A 1997; 94:10116-21.

18 Ang LC, Zhang Y, Cao L, et al. Versican enhances locomotion of astrocytoma cells and reduces cell adhesion through its G1 domain. J Neuropathol Exp Neurol 1999; 58:597-605.

19 Chen L, Yang BL, Wu Y, et al. G3 domains of aggrecan and PG$\mathrm{M} /$ versican form intermolecular disulfide bonds that stabilize cell-matrix interaction. Biochemistry 2003; 42:8332-41.

20 Yang BL, Cao L, Kiani C, et al. Tandem repeats are involved in G1 domain inhibition of versican expression and secretion and the G3 domain enhances glycosaminoglycan modification and product secretion via the complement-binding protein-like motif. J Biol Chem 2000; 275:21255-61.

21 Yang BL, Yang BB, Erwin M, et al. Versican G3 domain enhances cellular adhesion and proliferation of bovine intervertebral disc cells cultured in vitro. Life Sci 2003; 73:3399-413.

22 Zhang Y, Cao L, Kiani C, et al. Promotion of chondrocyte proliferation by versican mediated by G1 domain and EGF-like motifs. J Cell Biochem 1999; 73:445-57.

23 Iozzo RV, Naso MF, Cannizzaro LA, et al. Mapping of the versican proteoglycan gene (CSPG2) to the long arm of human chromosome 5 (5q12-5q14). Genomics 1992; 14:845-51.

24 Naso MF, Zimmermann DR, Iozzo RV. Characterization of the complete genomic structure of the human versican gene and functional analysis of its promoter. J Biol Chem 1994; 269:32999- 
3008.

25 McEver RP. Selectins. Curr Opin Immunol 1994; 6:75-84.

26 Dours-Zimmermann MT, Zimmermann DR. A novel glycosaminoglycan attachment domain identified in two alternative splice variants of human versican. J Biol Chem 1994; 269:32992-8.

27 Ito K, Shinomura T, Zako M, et al. Multiple forms of mouse PG-M, a large chondroitin sulfate proteoglycan generated by alternative splicing. J Biol Chem 1995; 270:958-65.

28 Zako M, Shinomura T, Ujita M, et al. Expression of PG-M(V3), an alternatively spliced form of PG-M without a chondroitin sulfate attachment in region in mouse and human tissues. J Biol Chem 1995; 270:3914-8.

29 Chen $\mathrm{L}, \mathrm{Wu} \mathrm{Y}$, Lee $\mathrm{V}$, et al. The folded modules of aggrecan G3 domain exert two separable functions in glycosaminoglycan modification and product secretion. J Biol Chem 2002; 277: 2657-65.

30 Kiani C, Lee V, Cao L, et al. Roles of aggrecan domains in biosynthesis, modification by glycosaminoglycans and product secretion. Biochem J 2001; 354:199-207.

31 Kiani $\mathrm{C}$, Chen L, Lee V, et al. Identification of the motifs and amino acids in aggrecan $\mathrm{G} 1$ and $\mathrm{G} 2$ domains involved in product secretion. Biochemistry 2003; 42:7226-37.

32 Kitagawa $\mathrm{H}$, Tsutsumi K, Tone $\mathrm{Y}$, et al. Developmental regulation of the sulfation profile of chondroitin sulfate chains in the chicken embryo brain. J Biol Chem 1997; 272:31377-81.

33 Laurent TC, Fraser JR. Hyaluronan. FASEB J 1992; 6:2397404

34 Toole BP. Hyaluronan and its binding proteins, the hyaladherins. Curr Opin Cell Biol 1990; 2:839-44

35 Bartolazzi A, Peach R, Aruffo A, et al. Interaction between CD44 and hyaluronate is directly implicated in the regulation of tumor development. J Exp Med 1994; 180:53-66.

36 Toole BP. Hyaluronan promotes the malignant phenotype. Glycobiology 2002; 12:37R-42R.

37 Itano N, Atsumi F, Sawai T, et al. Abnormal accumulation of hyaluronan matrix diminishes contact inhibition of cell growth and promotes cell migration. Proc Natl Acad Sci U S A 2002; 99: 3609-14.

38 Culty M, Nguyen HA, Underhill CB. The hyaluronan receptor (CD44) participates in the uptake and degradation of hyaluronan. J Cell Biol 1992; 116:1055-62.

39 Lee JY, Spicer AP. Hyaluronan: a multifunctional, megaDalton, stealth molecule. Curr Opin Cell Biol 2000; 12:581-6.

40 Bourguignon LY, Zhu H, Shao L, et al. Rho-kinase (ROK) promotes CD44v(3,8-10)-ankyrin interaction and tumor cell migration in metastatic breast cancer cells. Cell Motil Cytoskeleton 1999; 43:269-87.

41 Oliferenko S, Kaverina I, Small JV, et al. Hyaluronic acid (HA) binding to CD44 activates Rac1 and induces lamellipodia outgrowth. J Cell Biol 2000; 148:1159-64.

42 Zhu D, Bourguignon LY. Interaction between CD44 and the repeat domain of ankyrin promotes hyaluronic acid-mediated ovarian tumor cell migration. J Cell Physiol 2000; 183:182-95.

43 Bourguignon LY, Zhu $\mathrm{H}$, Chu A, et al. Interaction between the adhesion receptor, CD44, and the oncogene product, p185HER2, promotes human ovarian tumor cell activation. J Biol Chem 1997; 272:27913-8.

44 Ilangumaran S, Briol A, Hoessli DC. CD44 selectively associates with active Src family protein tyrosine kinases Lck and Fyn in glycosphingolipid-rich plasma membrane domains of human peripheral blood lymphocytes. Blood 1998; 91:3901-8.

45 Fitzgerald KA, Bowie AG, Skeffington BS, et al. Ras, protein kinase $\mathrm{C}$ zeta, and I kappa B kinases 1 and 2 are downstream effectors of CD44 during the activation of NF-kappa B by hyaluronic acid fragments in T-24 carcinoma cells. J Immunol 2000; 164:2053-63.

46 Noble PW, McKee CM, Cowman M, et al. Hyaluronan fragments activate an NF-kappa B/I-kappa B alpha autoregulatory loop in murine macrophages. J Exp Med 1996; 183:2373-8.

47 Koochekpour S, Pilkington GJ, Merzak A. Hyaluronic acid/ $\mathrm{CD} 44 \mathrm{H}$ interaction induces cell detachment and stimulates migration and invasion of human glioma cells in vitro. Int J Cancer 1995; 63:450-4.

48 Yamagata M, Saga S, Kato M, et al. Selective distributions of proteoglycans and their ligands in pericellular matrix of cultured fibroblasts. Implications for their roles in cell-substratum adhesion. J Cell Sci 1993; 106 (Pt 1):55-65.

49 Karvinen S, Kosma VM, Tammi MI, et al. Hyaluronan, CD44 and versican in epidermal keratinocyte tumours. Br J Dermatol 2003; 148:86-94.

50 Matsumoto K, Shionyu M, Go M, et al. Distinct interaction of versican/PG-M with hyaluronan and link protein. J Biol Chem 2003; 278:41205-12.

51 Yang BL, Zhang Y, Cao L, et al. Cell adhesion and proliferation mediated through the G1 domain of versican. J Cell Biochem 1999; 72:210-20.

52 Kawashima $\mathrm{H}$, Hirose $\mathrm{M}$, Hirose $\mathrm{J}$, et al. Binding of a large chondroitin sulfate/dermatan sulfate proteoglycan, versican, to L-selectin, P-selectin, and CD44. J Biol Chem 2000; 275:3544856.

53 Grumet M, Milev P, Sakurai T, et al. Interactions with tenascin and differential effects on cell adhesion of neurocan and phosphacan, two major chondroitin sulfate proteoglycans of nervous tissue. J Biol Chem 1994; 269:12142-6.

54 Salmivirta M, Elenius K, Vainio S, et al. Syndecan from embryonic tooth mesenchyme binds tenascin. J Biol Chem 1991; 266: 7733-39.

55 Erickson HP. Tenascin-C, tenascin-R and tenascin-X: a family of talented proteins in search of functions. Curr Opin Cell Biol 1993; 5:869-76.

56 Hagios C, Koch M, Spring J, et al. Tenascin-Y: a protein of novel domain structure is secreted by differentiated fibroblasts of muscle connective tissue. J Cell Biol 1996; 134:1499-512.

57 Weber P, Bartsch U, Rasband MN, et al. Mice deficient for tenascin-R display alterations of the extracellular matrix and decreased axonal conduction velocities in the CNS. J Neurosci 1999; 19:4245-62.

58 Margolis RU, Margolis RK. Chondroitin sulfate proteoglycans as mediators of axon growth and pathfinding. Cell Tissue Res 1997; 290:343-8.

59 Pesheva P, Probstmeier R, Spiess E, et al. Divalent Cations Modulate the Inhibitory Substrate Properties of Murine Gliaderived J1-160 and J1-180 Extracellular Matrix Glycoproteins for Neuronal Adhesion. Eur J Neurosci 1991; 3:356-65.

60 Yamaguchi Y. Lecticans: organizers of the brain extracellular matrix. Cell Mol Life Sci 2000; 57:276-89.

61 Forsberg E, Hirsch E, Frohlich L, et al. Skin wounds and severed nerves heal normally in mice lacking tenascin-C. Proc Natl Acad 
Sci U S A 1996; 93:6594-9.

62 Timpl R, Sasaki T, Kostka G, et al. Fibulins: a versatile family of extracellular matrix proteins. Nat Rev Mol Cell Biol 2003; 4: 479-89.

63 Olin AI, Morgelin M, Sasaki T, et al. The proteoglycans aggrecan and Versican form networks with fibulin-2 through their lectin domain binding. J Biol Chem 2001; 276:1253-61.

64 Isogai Z, Aspberg A, Keene DR, et al. Versican interacts with fibrillin-1 and links extracellular microfibrils to other connective tissue networks. J Biol Chem 2002; 277:4565-72.

65 Aspberg A, Adam S, Kostka G, et al. Fibulin-1 is a ligand for the C-type lectin domains of aggrecan and versican. J Biol Chem 1999; 274:20444-9.

66 Wight T. Versican: a versatile extracellular matrix proteoglycan in cell biology. Curr Opin Cell Biol 2002; 14:617.

67 Yamagata M, Yamada KM, Yoneda M, et al. Chondroitin sulfate proteoglycan (PG-M-like proteoglycan) is involved in the binding of hyaluronic acid to cellular fibronectin. J Biol Chem 1986; 261:13526-35.

68 Touab M, Villena J, Barranco C, et al. Versican is differentially expressed in human melanoma and may play a role in tumor development. Am J Pathol 2002; 160:549-57.

69 Braunewell KH, Pesheva P, McCarthy JB, et al. Functional involvement of sciatic nerve-derived versican- and decorin-like molecules and other chondroitin sulphate proteoglycans in ECMmediated cell adhesion and neurite outgrowth. Eur J Neurosci 1995; 7:805-14.

70 Sakko AJ, Ricciardelli C, Mayne K, et al. Modulation of prostate cancer cell attachment to matrix by versican. Cancer Res 2003; 63:4786-91.

71 Zheng PS, Wen J, Ang LC, et al. Versican/PG-M G3 domain promotes tumor growth and angiogenesis. FASEB J 2004; 18: 754-6.

72 Wijelath ES, Murray J, Rahman S, et al. Novel vascular endothelial growth factor binding domains of fibronectin enhance vascular endothelial growth factor biological activity. Circ Res 2002; 91:25-31.

73 Kawashima H, Atarashi K, Hirose M, et al. Oversulfated chondroitin/dermatan sulfates containing GlcAbeta1/IdoAalpha13 GalNAc(4,6-O-disulfate) interact with L- and P-selectin and chemokines. J Biol Chem 2002; 277:12921-30.

74 Hirose J, Kawashima H, Yoshie O, et al. Versican interacts with chemokines and modulates cellular responses. J Biol Chem 2001; 276:5228-34.

75 Pachynski RK, Wu SW, Gunn MD, et al. Secondary lymphoidtissue chemokine (SLC) stimulates integrin alpha 4 beta 7-mediated adhesion of lymphocytes to mucosal addressin cell adhesion molecule-1 (MAdCAM-1) under flow. J Immunol 1998; 161:952-6.

76 Kawashima H, Li YF, Watanabe N, et al. Identification and characterization of ligands for L-selectin in the kidney. I. Versican, a large chondroitin sulfate proteoglycan, is a ligand for L-selectin. Int Immunol 1999; 11:393-405.

77 Johnson PR. Role of human airway smooth muscle in altered extracellular matrix production in asthma. Clin Exp Pharmacol Physiol 2001; 28:233-6.

78 Bensadoun ES, Burke AK, Hogg JC, et al. Proteoglycan deposition in pulmonary fibrosis. Am J Respir Crit Care Med 1996; 154:1819-28.
79 Borland G, Ross JA, Guy K. Forms and functions of CD44. Immunology 1998; 93:139-48.

80 Aruffo A, Stamenkovic I, Melnick M, et al. CD44 is the principal cell surface receptor for hyaluronate. Cell 1990; 61:1303-13.

$81 \mathrm{Yu}$ Q, Toole BP, Stamenkovic I. Induction of apoptosis of metastatic mammary carcinoma cells in vivo by disruption of tumor cell surface CD44 function. J Exp Med 1997; 186:1985-96.

82 Vink J, Thomas L, Etoh T, et al. Role of beta-1 integrins in organ specific adhesion of melanoma cells in vitro. Lab Invest 1993; 68:192-203.

83 Lesley J, Howes N, Perschl A, et al. Hyaluronan binding function of CD44 is transiently activated on T cells during an in vivo immune response. J Exp Med 1994; 180:383-7.

84 Gunthert U, Hofmann M, Rudy W, et al. A new variant of glycoprotein CD44 confers metastatic potential to rat carcinoma cells. Cell 1991; 65:13-24.

85 Sy MS, Guo YJ, Stamenkovic I. Distinct effects of two CD44 isoforms on tumor growth in vivo. J Exp Med 1991; 174:859-66.

86 Hua Q, Knudson CB, Knudson W. Internalization of hyaluronan by chondrocytes occurs via receptor-mediated endocytosis. J Cell Sci 1993; 106(Pt 1):365-75.

87 Varner JA, Cheresh DA. Integrins and cancer. Curr Opin Cell Biol 1996; 8:724-30.

88 Giancotti FG, Ruoslahti E. Integrin signaling. Science 1999; 285: 1028-32.

89 Denda S, Reichardt LF, Muller U. Identification of osteopontin as a novel ligand for the integrin alpha8 beta1 and potential roles for this integrin-ligand interaction in kidney morphogenesis. Mol Biol Cell 1998; 9:1425-35.

90 Brandenberger R, Schmidt A, Linton J, et al. Identification and characterization of a novel extracellular matrix protein nephronectin that is associated with integrin alpha8betal in the embryonic kidney. J Cell Biol 2001; 154:447-58.

91 Yokosaki Y, Matsuura N, Higashiyama S, et al. Identification of the ligand binding site for the integrin alpha9 betal in the third fibronectin type III repeat of tenascin-C. J Biol Chem 1998; 273:11423-8.

$92 \mathrm{Wu} \mathrm{Y,} \mathrm{Chen} \mathrm{L,} \mathrm{Zheng} \mathrm{PS,} \mathrm{et} \mathrm{al.} \mathrm{beta} \mathrm{1-Integrin-mediated} \mathrm{glioma}$ cell adhesion and free radical-induced apoptosis are regulated by binding to a C-terminal domain of PG-M/versican. J Biol Chem 2002; 277:12294-301.

93 Yamada KM, Even-Ram S. Integrin regulation of growth factor receptors. Nat Cell Biol 2002; 4:E75-6.

94 Schwartz MA, Assoian RK. Integrins and cell proliferation: regulation of cyclin-dependent kinases via cytoplasmic signaling pathways. J Cell Sci 2001; 114:2553-60.

95 Frisch SM, Screaton RA. Anoikis mechanisms. Curr Opin Cell Biol 2001; 13:555-62.

96 Lu Z, Jiang G, Blume-Jensen P, et al. Epidermal growth factorinduced tumor cell invasion and metastasis initiated by dephosphorylation and downregulation of focal adhesion kinase. Mol Cell Biol 2001; 21:4016-31.

97 Wu Y, Chen L, Cao L, Sheng W, Yang BB. Overexpression of the C-terminal PG-M/versican domain impairs growth of tumor cells by intervening in the interaction between epidermal growth factor receptor and beta1-integrin. J Cell Sci 2004; 117:2227-37.

$98 \mathrm{Wu}$ Y, Sheng W, Chen L, et al. Versican V1 isoform induces neuronal differentiation and promotes neurite outgrowth. Mol Biol Cell 2004; 15:2093-104. 
99 Pouyani T, Seed B. PSGL-1 recognition of P-selectin is controlled by a tyrosine sulfation consensus at the PSGL-1 amino terminus. Cell 1995; 83:333-43.

100 Sako D, Chang XJ, Barone KM, et al. Expression cloning of a functional glycoprotein ligand for P-selectin. Cell 1993; 75:117986.

101 Moore KL, Patel KD, Bruehl RE, et al. P-selectin glycoprotein ligand-1 mediates rolling of human neutrophils on P-selectin. J Cell Biol 1995; 128:661-71.

102 Leppanen A, Penttila L, Renkonen O, et al. Glycosulfopeptides with O-glycans containing sialylated and polyfucosylated polylactosamine bind with low affinity to P-selectin. J Biol Chem 2002; 277:39749-59.

103Zheng PS, Vais D, Lapierre D, et al. PG-M/versican binds to Pselectin glycoprotein ligand-1 and mediates leukocyte aggregation.
J Cell Sci 2004; 117:5887-95.

104Olsson U, Camejo G, Hurt-Camejo E, et al. Possible functional interactions of apolipoprotein B-100 segments that associate with cell proteoglycans and the ApoB/E receptor. Arterioscler Thromb Vasc Biol 1997; 17:149-55.

105Olin KL, Potter-Perigo S, Barrett PH, et al. Lipoprotein lipase enhances the binding of native and oxidized low density lipoproteins to versican and biglycan synthesized by cultured arterial smooth muscle cells. J Biol Chem 1999; 274:34629-36.

106 Srinivasan SR, Xu JH, Vijayagopal P, et al. Low-density lipoprotein binding affinity of arterial chondroitin sulfate proteoglycan variants modulates cholesteryl ester accumulation in macrophages. Biochim Biophys Acta 1995; 1272:61-7.

Editor: Dr. Yufang SHI 